\title{
Enseñanza de construcción de estructuras para arquitectos mediante simulación 3D.
}

\section{Teaching of construction of structures for architects through 3D simulation.}

\author{
Cubillos, Sonia ${ }^{1} \&$ López, Francisco ${ }^{2}$ \\ ${ }^{1}$ Universidad La Gran Colombia, Bogotá, Colombia. \\ ${ }^{2}$ Universidad Nacional de Colombia, Bogotá, Colombia. \\ ${ }^{1}$ sonia.cubillos@ugc.edu.co / https://orcid.org/0000-0002-5513-2831 \\ 2fglopezp@unal.edu.co / https://orcid.org/0000-0001-5695-9905 \\ Recibido el 09 de abril de 2021, aprobado el 18 de junio de 2021
}

RESUMEN | El Modelado de Información de la Construcción (BIM) es una metodología basada en sistemas digitales aplicados a la arquitectura y la construcción, generando la creación de nuevas formas de trabajo para la coordinación técnica de los proyectos.

Este sistema se puede aplicar para la enseñanza-aprendizaje de los estudiantes de arquitectura, para identificar y resolver los problemas entre los diferentes componentes de un proyecto. Se diseñó una metodología educativa de acuerdo con la enseñanza en el proceso de construcción estructural, utilizando modelos de simulación 3D para la comprensión de los sistemas que integran el proyecto, permitiendo simular los diferentes componentes de un sistema estructural: excavaciones, cimentaciones, vigas y losas y anticipar la relación con el espacio arquitectónico.

La metodología se aplicó para el proceso de enseñanza, donde los estudiantes pudieron comprender de una manera integradora la relación entre los componentes estructurales, su proceso constructivo y su relación con el espacio arquitectónico.

PALABRAS CLAVE | Calidad educativa, construcción deestructuras, simulación, integración de sistemas

ABSTRACT | Building Information Modeling (BIM) is a methodology in advancement in digital systems applied on architecture and construction, generating the creation of new ways of working for the technical coordination of projects.

This system can be applied for the teaching/learning of the architecture students, in order to identify and solve the different issues and elements of a project. An educational methodology was designed in accordance with the teaching of the structural construction process, using 3D simulation models to understand the elements comprising the project, allowing the simulation 
of the different components of a structural system; excavation, foundation, slabs and beams and anticipate de relationship with the architectural space.

The methodology was used in the teaching process, where the students were able to understand the relationship among the structural components in comprehensive way, its building process and its relationship with the architectural space.

KEYWORD | Educational quality, construction of structures, simulation, integration systems

\section{Introducción}

Los procesos de enseñanza aprendizaje con el uso de medios tecnológicos que parten de la simulación digital estimula la participación y genera una mayor comunicación conceptual entre los estudiantes de acuerdo con Guitert, \& Giménez, (2005) mediante la anticipación y verificación con el software, facilitando la interacción entre el proyecto arquitectónico y los componentes técnicos, mediante la utilización de metodologías basadas en la solución de problemas que faciliten la integración de las diferentes áreas que intervienen en el proyecto. La observación de las diferencias y similitudes entre el mundo digital y el mundo real, genera en el estudiante una reelaboración de conceptos, según López et al. (2006), que le acercan al contexto real del proyecto.

La simulación del proceso constructivo en este contexto se concibe como herramienta de apoyo, favorece el aprendizaje por descubrimiento, obliga a demostrar lo aprendido, permite la ejercitación del estudiante de forma independiente, favorece los procesos de reproducción de la experiencia permite control de variables, y facilita la autoevaluación (Cabero J. \& Costas J. 2017).

$\mathrm{BIM}^{1}$ facilita los procesos de aproximación digital para el desarrollo de proyectos arquitectónicos, y se convierten en un medio que hace posible una comprobación de los modelos estructurales, igualmente permiten las modificaciones en tiempo real, integrando elementos paramétricos en estructuras arquitectónicas como indica Andrade (2020) esto facilita las explicaciones estructurales de diseños de acuerdo con Sampaio, A.Z. \& Gomes, A.M. (2021) proporcionan métodos para la verificación de las estructuras, además de proveer elementos de programación, control y costos del proyecto.

La enseñanza de los procesos constructivos de estructuras en la carrera de Arquitectura está en estrecha correspondencia con el aprendizaje de conceptos técnicos como lo expresan Merritt \& Ricketts (1997), desde varias categorías: Regulación (normas y reglamentos). Lugar (estudio geotécnico, topografía, humedad y salinidad). Uso, (condiciones de uso, configuración arquitectónica), Diseño estructural (estudio de cargas, materiales, resistencias, configuración geométrica, sistema estructural), Métodos y equipos (proceso constructivo, fases, secuencias de actividades, componentes constructivos), además con los sistemas técnicos que intervienen en el proyecto arquitectónico como son los sistemas eléctricos, sanitarios, ambientales, hidráulicos, entre otros.

La enseñanza de los procesos constructivos en el campo de la arquitectura, tradicionalmente se realiza con exposiciones orales de los conceptos teóricos fundamentales, por parte de los docentes; donde el estudiante se convierte en un receptor de la información impartida. Generalmente, se

1 "Conjunto de metodologías, tecnologías y estándares que permiten diseñar, construir y operar una edificación o infraestructura de forma colaborativa en un espacio virtual." adoptado en base a la definición de Bilal Succar por Plan BIM Chile. 
espera que los estudiantes manifiesten los conceptos teóricos impartidos por los docentes, mediante la elaboración de planos en dos dimensiones, donde ésta forma de representación, la cual está configurada por símbolos abstractos, no es clara, la comunicación de la información esta fraccionada y no es sistémica, al no encontrarse integrados en un solo plano los diferentes componentes y sistemas que intervienen en el proyecto arquitectónico como lo expresa Arrieta (2018).

La complejidad de los procesos de enseñanza - aprendizaje desde la perspectiva de la integración de sistemas constructivos, se dificulta debido a que implica la adquisición y asimilación significativa de conceptos y procedimientos de los siguientes aspectos:

a) El estudiante debe comprender, visualizar e imaginar las relaciones entre las diferentes redes (hidráulicas eléctricas y estructurales, entre otras).

b) El estudiante estará en capacidad de comprender, visualizar y representar bidimensional y tridimensionalmente los procesos de construcción de las estructuras a nivel de detalles en cada etapa constructiva, tanto de los elementos estructurales como de otros elementos arquitectónicos.

c) El estudiante estará en capacidad de establecer las posibles colisiones entre los diferentes sistemas y/o redes constructivas, que puedan afectar la calidad constructiva en la obra.

d) El estudiante estará en capacidad establecer las especificaciones y particularidades acordes con las normas constructivas vigentes. Por todo lo anterior la asimilación de los conceptos, procedimientos y relaciones estructurales, y arquitectónicos, desde la comprensión de lo constructivo como un todo, se dificulta en la mente del estudiante.

Por otra parte, la evaluación del aprendizaje del proceso constructivo, tradicionalmente se ha realizado mediante correcciones sucesivas de los planos bidimensionales elaborados por los estudiantes; donde el docente demuestra en forma explícita su conocimiento y experiencia; sin dar lugar a la intervención activa del estudiante; lo cual se constituye como una metodología, que no favorece la construcción, reelaboración y generación de propuestas que permitan a los estudiantes plantear y adquirir experiencias en torno a procesos de resolución de problemas referentes al aprendizaje de procesos constructivos, según Jonassen D. (2000) y Honebein P \& Reigeluth Charles M. (2020). En este sentido en el diseño de simuladores para la formación constituye uno de modelos más relevantes el propuesto por Jonassen (2000), denominado "Entornos de aprendizaje constructivista" (EAC), el cual continúa siendo vigente en la actualidad (Cabero J. \& Costas J. 2017).

Por lo tanto, se puede inferir que los procesos de enseñanza aprendizaje tradicional, en el área tanto de estructura como de construcción en general, no han promovido en los estudiantes el desarrollo de habilidades, y criterios adecuados necesarios para la solución de problemas (Rodríguez, C. \& Fernández-Batanero, J. 2017); debido en parte a la gran complejidad que demanda la comprensión significativa de los conceptos, procedimientos y habilidades implicados en la integración y coordinación de un proyecto arquitectónico. En este contexto cabe resaltar la importancia de lograr proyectos arquitectónicos funcionales de calidad estructural con métodos constructivos adecuados acorde a lo expresado por Orbe M, Toledo J \& Ávila M. (2021).

Debido a la problemática anteriormente expuesta, se planteó la hipótesis, de que la enseñanza mediada por medios digitales apoyados en la simulación de procesos constructivos de una estructura, promueve el aprendizaje significativo del sistema constructivo, con sus respectivas interrelaciones entre los diversos componentes arquitectónicos y técnicos, sus fases, procedimientos y posibles 
colisiones entre las diferentes redes; permitiéndole al estudiante la comprensión, percepción y desarrollo de habilidades de simulación tridimensional implicados en la concepción, creación, interpretación y solución de problemas propios del proyecto arquitectónico.

El aprendizaje significativo en este contexto según López et al (2012) manifiestan lo siguiente:

En este caso, se define aprendizaje significativo, como aquel que promueve la compresión de los procesos de integración de los diferentes sistemas que intervienen en el proyecto de arquitectura en los estudiantes, la coordinación eficiente y eficaz los procesos constructivos referentes a todo tipo de instalaciones técnicas que intervienen en un proyecto arquitectónico, teniendo en cuenta las normativas, materiales y detalles constructivos, y que finalmente, permita generar propuestas arquitectónicas simulando los procesos constructivos mediante el uso de medios digitales, para la gestión de la calidad en el proyecto (p. 2).

La hipótesis de trabajo de la propuesta formulada, plantea un cambio metodológico cuyo objetivo es el de promover la compresión de los procesos constructivos, haciendo un mayor énfasis en el aprendizaje profundo, que permite al estudiante además de desarrollar la capacidad para describir los componentes, materiales y especificaciones (elementos focales de la enseñanza tradicional), proponer y solucionar problemas mediante la utilización de medios digitales, generando procesos de investigación que favorece la integración de todos los sistemas implicados en la ejecución constructiva del proyecto arquitectónico, el cual se sintetiza en simulaciones tridimensionales detalladas, que facilitan al docente los procesos evaluativos debido a la claridad de la visualización de los medios.

La aplicación de esta metodología se realizó en el curso de Construcciones de Baja Complejidad, de la carrera de Arquitectura de la Escuela de Arquitectura y Urbanismo, Universidad Nacional de Colombia, con dos grupos diferentes con un número de veinte (20) estudiantes por grupo.

Uno de los grupos trabajó de forma tradicional (grupo testigo), dicho grupo planteó las respuestas en forma tradicional a partir de resolver un problema específico en dos dimensiones (planos técnicos constructivos tradicionales). El otro grupo, aplicando la nueva metodología (grupo gestor), planteó las respuestas al mismo problema, generando la información técnica constructiva en modelos tridimensionales.

\section{Metodología}

La metodología del aprendizaje propuesto se fundamentó en experiencias con cursos anteriores (López \& Cubillos 2011), dichas experiencias fueron aplicadas en el curso Construcciones de Alta Complejidad del primer semestre de 2020, y en el curso de Construcciones de Mediana Complejidad del segundo semestre de 2020, los pasos metodológicos para la nueva aplicación se revisaron y ajustaron de acuerdo a los contenidos temáticos de la asignatura los cuales se presentan a continuación:

a) Identificaron los conocimientos previos de los estudiantes partiendo de la aplicación de una evaluación diagnóstica inicial. Para la identificación de los conocimientos previos se definieron tres categorías de evaluación, la primera sobre los materiales de construcción, la segunda sobre los procesos constructivos y la tercera sobre los procesos de control de calidad en la construcción.

b) Se ajustó la metodología buscando coherencia entre los objetivos del curso, con los de la visión y misión de la Universidad, los de la Facultad, los de la carrera de arquitectura y los propios 
del área de tecnología. Coherencia entre lo que se espera, se enseña y se evalúa acorde con Biggs, J. (2006) en su modelo pedagógico de alineamiento constructivo. El alineamiento constructivo es un enfoque planteado por Biggs, en donde el aprendizaje del estudiante y los procesos didácticos de enseñanza guardan coherencia con la evaluación del aprendizaje, esto ha sido denominado, alineación constructiva del aprendizaje y busca promover el aprendizaje profundo (aprendizaje significativo en el estudiante), se basa en dos principios del constructivismo: el aprendizaje y el alineamiento de la enseñanza.

c) Diseño y elaboración de un modelo didáctico de la estructura de una construcción de baja complejidad (vivienda unifamiliar en dos pisos con niveles, de no más de 120 metros cuadrados, construcción aporticada en concreto).

d) Aplicación del modelo didáctico, explicitando los conceptos estructurales, donde se hacen relevantes las relaciones entre el modelo estructural y su afectación al proyecto arquitectónico. Diseño del proyecto arquitectónico. Condiciones del lugar. Aplicación de las normativas. Diseño estructural. Proceso constructivo de la estructura de la edificación. (Figura 1).

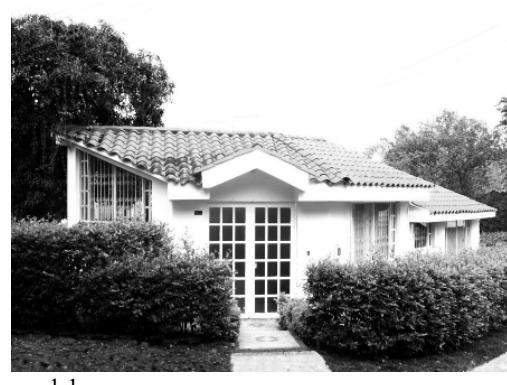

1.1

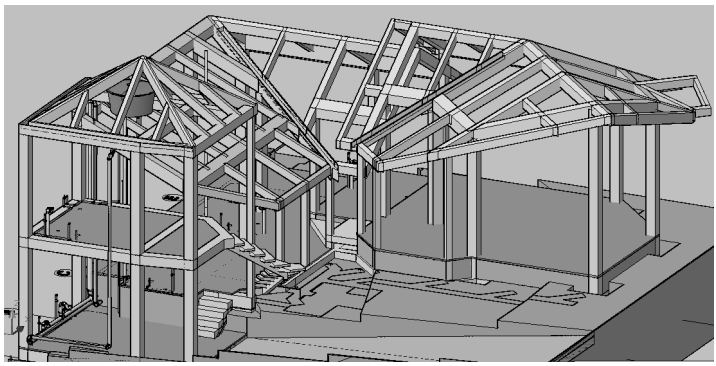

1.4

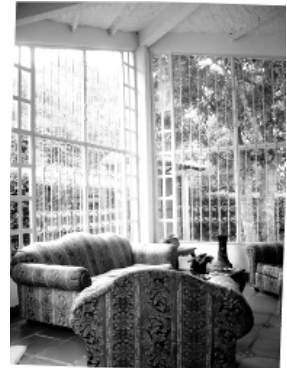

1.2

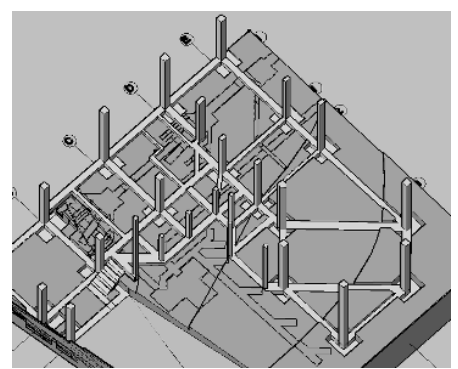

1.3

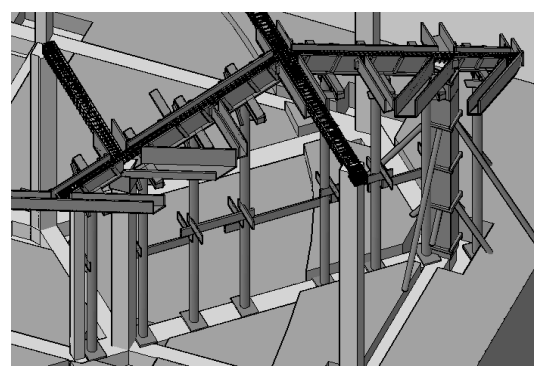

1.5

Figura 1: Proyecto arquitectónico ubicado en Nocaima, Cundinamarca (Colombia). Se tuvieron en cuenta las condiciones normativas de la Alcaldía, la norma antisísmica de Colombia NSR-10 y todas las normas constructivas colombianas. El numeral 1.1 muestra el proyecto arquitectónico existente desde el exterior, el numeral 1.2 muestra el proyecto existente desde el interior. El numeral 1.3 muestra el sistema de cimentación. El numeral 1.4 muestra la estructura aporticada en concreto. El numeral 1.5 muestra el sistema de encofrado para fundir el concreto.

Fuente: Elaborado por los autores.

Igualmente, otro objetivo de aprendizaje se planteó desde la coordinación técnica del proceso constructivo y la integración de sistemas técnicos (para el proyecto se escogieron el de suministro hidráulico y el de evacuación sanitario) que intervienen en el proyecto arquitectónico.

Los objetivos planteados anteriormente permitían al estudiante identificar las posibles colisiones entre los sistemas y como su planteamiento puede afectar el diseño arquitectónico, el diseño estructural, el proceso constructivo y adicionalmente le permite conocer los componentes de los sistemas seleccionados, al igual que el conocimiento de los materiales a escoger, sus tipos de unión, 
direccionamiento y por lo tanto la definición de las cantidades (Figura 2).

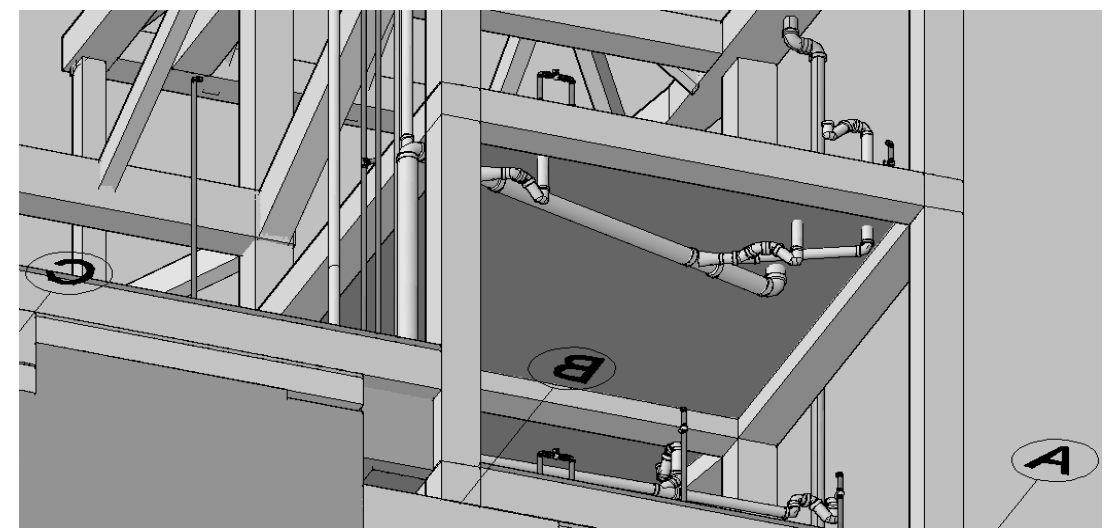

Figura 2: Se diseñó el proyecto estructural teniendo en cuenta la integración con el proyecto arquitectónico y los diferentes sistemas técnicos que intervienen en la vivienda, respondiendo a las condiciones del lugar (topografía, clima, localización, etc.). Fuente: Elaborado por los autores.

La unidad didáctica se presentó en forma digital mostrando la secuencia constructiva, de manera que los estudiantes y los docentes tuviesen un apoyo en los procesos de enseñanza aprendizaje.

e) Selección del software, la escogencia se hizo teniendo en cuenta la facilidad de adquisición por parte de los estudiantes y el tipo de hardware con que contaban (algunos decidieron trabajar con Revit y otros con Archicad). Se analizó el potencial del medio para lograr una mejor aproximación al conocimiento y el desarrollo de capacidades de resolución de problemas por parte de los estudiantes.

f) Definición de grupos. En el primer grupo (grupo testigo), el proceso de enseñanza se desarrolló de forma tradicional, el análisis, la elaboración y presentación de trabajos se hicieron de la forma convencional (correcciones sucesivas del docente, sobre planos bidimensionales construidos por los estudiantes). En el segundo grupo (grupo gestor), la enseñanza se realizó apoyada en el modelado, trabajando con los medios de simulación, el análisis, la elaboración y las respuestas se dieron a través de modelos en 3D.

g) Definición de la situación o ejercicio a desarrollar en ambos grupos, acorde al alcance en tiempo, recursos, conocimientos de las estudiantes y del programa académico (Figura 3).

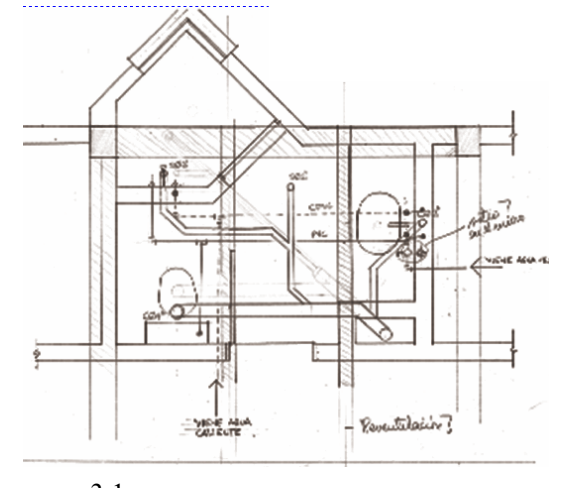

3.1

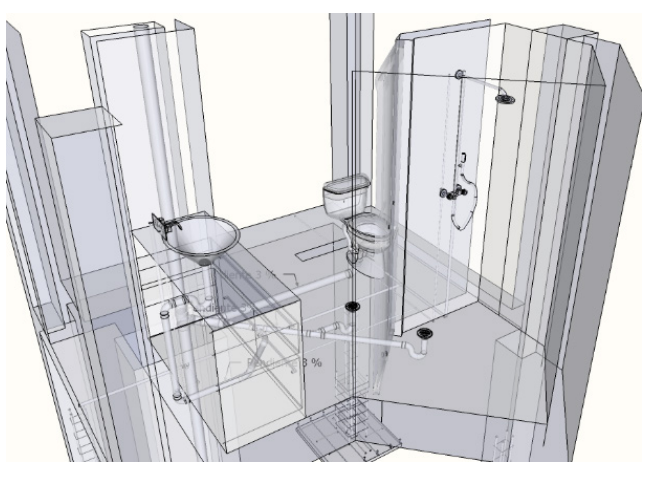

3.2

Figura 3: Fases del ejercicio desarrollado en el grupo gestor. La imagen de la izquierda (3.1) representa el plano bidimensional elaborado por un estudiante del grupo testigo, a la derecha (3.2) la simulación tridimensional del ejercicio realizada por un estudiante del grupo gestor, donde se muestra parte del proceso constructivo, como se observa es muy difícil definir y entender tanto detalle observando solo la imagen de la izquierda. Fuente: Elaborado por los autores. 
h) Se estableció la forma de presentación de la información del proceso y de los resultados. Se diseño un formato que permitiese al estudiante presentar toda la información analizada en el modelo 3D, donde se apreciara la integración de los sistemas y componentes que intervienen en el proyecto.

El diseño del formato fue organizado en forma de matriz, en donde se definieron los criterios que el estudiante debía tener en cuenta la identificación, descripción y análisis de las fases del proceso constructivo y de los diversos sistemas integrados que intervienen en el proyecto. Elaborado por los autores (Figura 4).

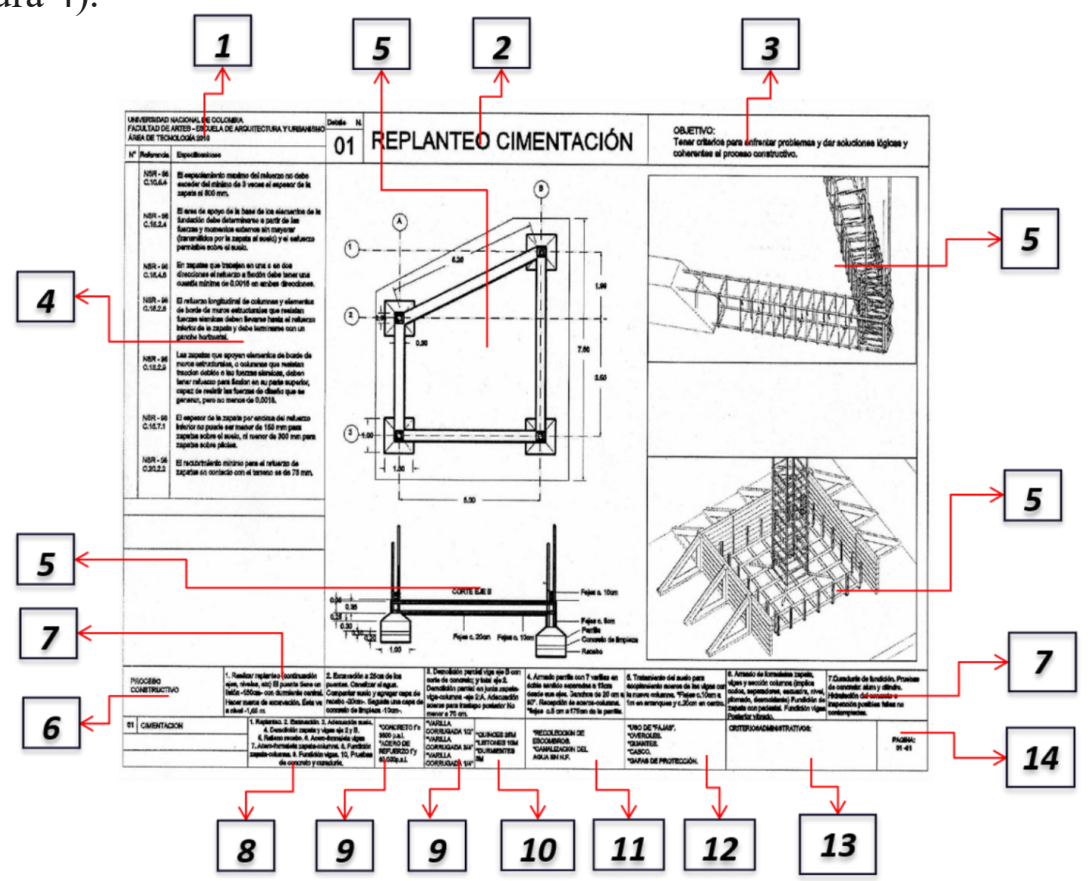

Figura 4: Formato de presentación de resultados. Leyenda (1)Institución, (2)Temática, (3)Objetivo,(4) Normativa,(5) Contenido técnico gráfico, (6)Proceso constructivo, (7) Secuencia y descripción del proceso constructivo, (8) Enumeración de la secuencia constructiva, (9) Equipo y maquinaria, (10) Materiales, (11) Manejo ambiental, (12) Seguridad y salud ocupacional, (13) Criterios administrativos, (14) Número de plano. Fuente: Elaborado por los autores.

i) Identificación y comparación de los procesos desarrollados por los dos grupos (Figura 5).

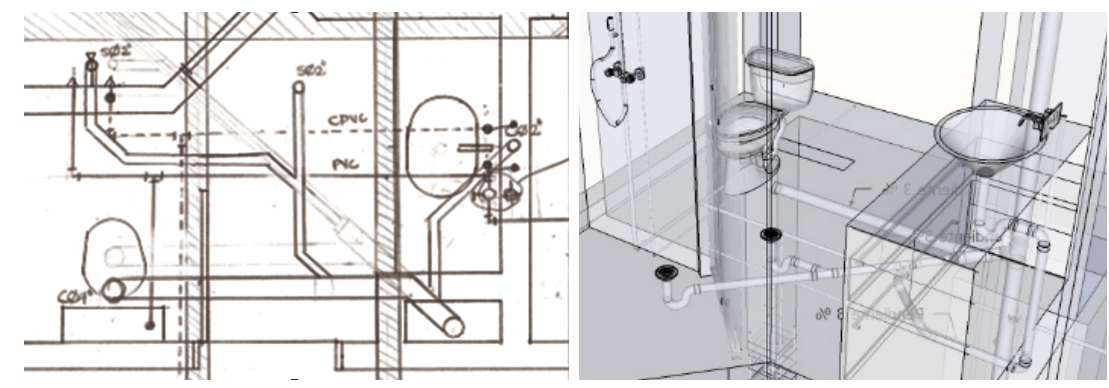

Figura 5: Comparación de resultados entre el grupo testigo y el grupo gestor (detalles ampliados de la figura 3.1 y 3.2). La diferencia entre la imagen izquierda (grupo testigo) y la imagen derecha (grupo gestor), permite corroborar que los estudiantes del grupo gestor deben efectuar un mayor esfuerzo cognitivo, permitiéndole al docente una visualización y valoración del aprendizaje; en contraste en el grupo testigo, el docente no puede comprobar los procesos de aprendizaje obtenidos por los estudiantes, como se ha hecho tradicionalmente mediante planos bidimensionales. Fuente: Resultado de los estudinates. 
j) Definición de criterios evaluativos mediante el análisis de las respuestas que permitan medir adecuadamente los resultados (Figura 6). Se diseñó una matriz de evaluación, estableciendo los criterios de aprendizaje a los cuales debía llegar el estudiante, dicha matriz se aplicó a ambos grupos (grupo testigo y grupo gestor).

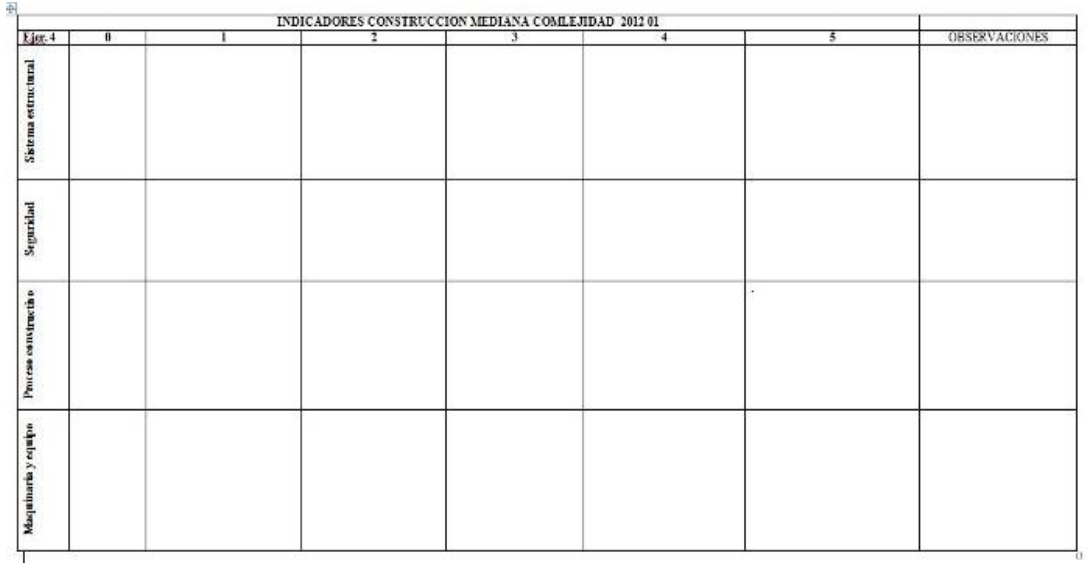

Figura 6: Rúbrica de evaluación. Se establecieron criterios de evaluación aplicados a los dos grupos (grupo testigo y grupo gestor) para los ejercicios, mediante un formato tipo matriz, con el fin de poder comparar los procesos de aprendizaje de ambos grupos. Fuente: Elaborado por los autores.

La evaluación tradicional de correcciones sucesivas de planos bidimensionales se sustituye por la evaluación fundamentada en la simulación tridimensional, que permita construir y visualizar los procesos de coordinación técnica de los diferentes sistemas que intervienen en el proceso constructivo; analizando las interrelaciones entre la estructura y los diferentes sistemas y sus posibles conflictos e integrando en un todo las diversas áreas que intervienen en el proyecto arquitectónico.

\section{Resultados}

En el grupo testigo, los estudiantes no manifestaron con claridad, la integración de sistemas que inciden el proyecto arquitectónico, tampoco se demostró los procesos constructivos, cuya teoría fue enseñada en el aula, solo presentaron adecuadamente los planos técnicos, más no las fases y secuencias que establezcan cuales son los procedimientos, usualmente teniendo la intención de plantear una aproximación al proceso, genera respuestas sin criterio.

Por otra parte, las respuestas dadas por los estudiantes del grupo testigo, sobre procesos constructivos, carecen de fundamentación científica.

El grupo testigo manifestó en sus trabajos las siguientes características: a) Se concentró en la solución específica de los componentes. b) Tuvo en cuenta el origen de las interacciones entre estructura y proyecto arquitectónico. c) Buscó el desarrollo de los detalles constructivos en dos dimensiones. d) Modificaba un componente a la vez sin integrar con los otros sistemas que afectan el proyecto. e) No validaron la teoría, solo se remitieron a plantear respuestas con los planos bidimensionales.

El grupo gestor manifestó las siguientes características: a) Encontró las interacciones entre los elementos que componen el proyecto. b) Tuvieron en cuenta las afectaciones al proyecto arquitectónico que causarían cambios en la estructura. c) Buscó el desarrollo en tres dimensiones de los sistemas teniendo en cuenta su interacción. d) Cuando modificaba un componente de la estructura, identificaba la afectación a los otros sistemas, modificando los mismos. e) Validaron la 
teoría a partir del modelo tridimensional, referenciándolo con la realidad del proyecto construido.

La simulación en 3D, demandó a los estudiantes del grupo gestor, investigar diversas alternativas para lograr dar respuestas adecuadas a las situaciones y problemas del proyecto arquitectónico, que en la metodología tradicional no se planteaban; esto les permitió a los educandos integrar los diversos componentes y fases, hasta obtener una planificación de la ejecución del proyecto constructivo viable mediante el desarrollo de soluciones detalladas. (Figura 7 y Figura 8).
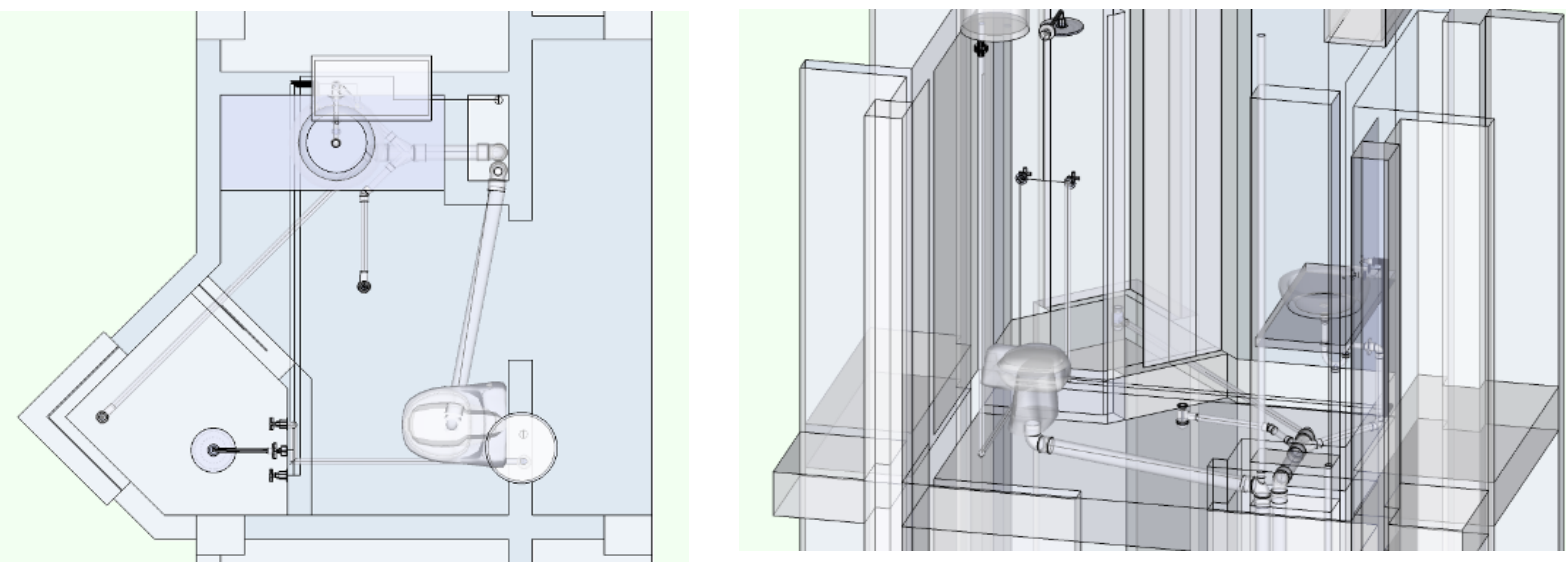

Figura 7: Simulación tridimensional de las fases del proceso. Las imágenes superiores representan las condiciones estructurales, y como el estudiante tuvo en cuenta la integración entre estructura e instalaciones técnicas. Fuente: Resultados de los estudiantes.

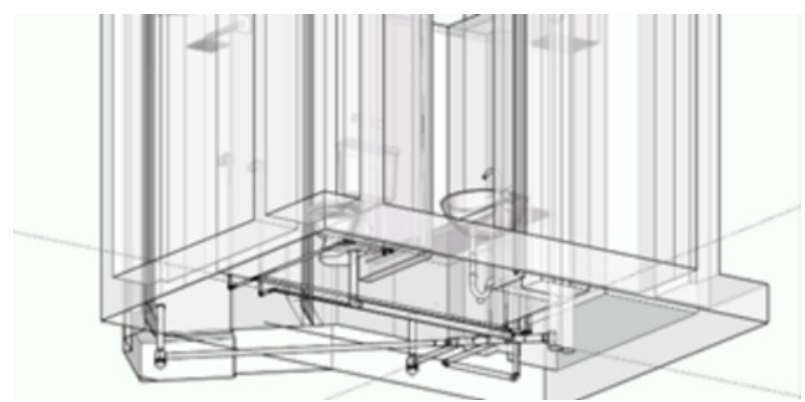

Figura 8: Simulación tridimensional de los componentes integrados con el sistema estructural. La imagen superior representan los componentes técnicos hidrosanitarios y su afectación con la estructura, son perceptibles los errores que comete el estudiante, pero se aprecia el esfuerzo por definir todos los elementos que intervienen en el proyecto, inclusive con sus especificaciones técnicas. Fuente: Resultados de los estudiantes.

\section{Conclusiones}

La aplicación de la metodología de instrucción propuesta permitió la mejora los siguientes aspectos:

La comunicación del proceso constructivo mejoró notoriamente respecto a la metodología tradicional, debido a que la visualización tridimensional favorece la comprensión espacial de los elementos estructurales y en general de los procesos constructivos, permitiendo al estudiante comprender los detalles y particularidades de cada uno de los diferentes componentes del proyecto.

La metodología tridimensional favoreció la construcción del conocimiento por interacción entre el entorno tridimensional y los conceptos previos del individuo que aprende: promoviendo el desarrollo de la autonomía y regulación en los estudiantes, esto permitió la asimilación significativa 
de conceptos de alta complejidad, debido a procesos de interacción entre el entorno digital 3D y el estudiante; lo cual potencializó las estrategias cognitivas tridimensionales enriqueciendo la visualización, percepción y comunicación de los diversos sistemas.

El desarrollo creación y solución del proyecto constructivo mediante simulación 3D permitió a los estudiantes: Incrementar la capacidad de identificación, creación y anticipación de las fases del proceso constructivo; mediante la generación de una "memoria digital", que les permitió la identificación de conflictos entre las diferentes redes, y por tanto la solución adecuada de los mismos.

Aumentar la motivación intrínseca en los estudiantes, ya que no buscaban una recompensa externa (calificación), mostrando el interés de trabajar en función del aprendizaje.

La enseñanza apoyada en la solución de problemas equivalentes a la práctica profesional crea una gran motivación en el estudiante, ya que se aproximación en forma directa a la construcción del proyecto, generando habilidades cognitivas en entornos de aprendizaje constructivista, de acuerdo Espinoza M. C. (2016).

El desarrollo creación y solución del proyecto constructivo mediante simulación 3D permitió a los docentes:

- La identificación de conflictos conceptuales en los procesos constructivos propuestos por los estudiantes, con una mayor claridad.

- Generar procesos de evaluación integrales, en los cuales la corrección del proyecto se asimila o identifica con los procesos reales demandados en el ámbito profesional.

Debido a la complejidad de los procesos evaluativos, se hizo necesario correcciones en videoproyector donde todo el grupo visualizaba los errores y aciertos de los proyectos de sus compañeros con el fin de extrapolarlos a los demás proyectos, debido a la excesiva demanda de tiempo del docente que exigió esta metodología.

La implementación de esta metodología de simulación tridimensional permitió mejorar el conocimiento conceptual, procedimental, condicional y funcional de los procesos de construcción estructural en los estudiantes los cuales se enunciarán a continuación:

Conocimiento Conceptual: Los estudiantes comprendieron de una forma más profunda la integración de los diferentes sistemas en el proyecto, lo cual les permitió establecer criterios para crear y proponer estructuras viables, acordes a los espacios del diseño, lo cual redunda en el ahorro de tiempo e inversión durante la construcción.

Los estudiantes desarrollaron criterios que les permitieron tomar decisiones óptimas, y anticiparse a los problemas propios del diseño de estructura, que solo sería posible adquirir con muchos años de experiencia profesional.

Conocimiento Procedimental: Los estudiantes asimilaron mediante la construcción de simulaciones digitales, los pasos y procedimientos necesarios para la construcción de estructuras, representándolos en forma detallada, lo cual les permitió adquirir habilidades no solo constructivas, sino también el desarrollo de capacidades de comunicación digital.

Conocimiento Condicional: Los estudiantes durante los procesos de simulación tridimensional lograron percibir colisiones, y problemas de adaptación de las estructuras a los espacios de diseño, 
lo cual les permitió anticiparse a los posibles problemas en obra, corrigiendo y adaptando la estructura al diseño, esto facilito las adquisición y asimilación de conocimiento procedimental relacionado a saber cómo y cuándo es necesario modificar las estructuras acordes al tipo de diseño arquitectónico propuesto.

Conocimiento Funcional: La metodología propuesta permitió a los estudiantes tener un conocimiento de carácter más global donde se percibido y comprendió las relaciones entre los componentes estructurales y las demás redes constructivas del proyecto, lo cual permite la creación de criterios globalizadoras amplios aplicables a variados contextos constructivos, debido a la discusión y evaluación publica de los diversos proyectos de cada uno de los estudiantes.

El software fue utilizado como una plataforma, de visualización, que facilita y promueve los procesos de comprensión profunda, comprobación de hipótesis constructivas y ejecución de procesos de construcción, favoreciendo la integración óptima de los diversos sistemas.

En síntesis, el desarrollo de la metodología de simulación 3D en entornos digitales para la enseñanza y el aprendizaje de procesos constructivos referidos a la construcción de estructuras, favoreció la adquisición de un conocimiento más profundo por parte del estudiante; conocimiento enriquecido por los diversos conceptos, procedimientos y funcionamiento detallado subyacente al diseño arquitectónico.

El uso y aplicación de la simulación en 3D, favoreció la comprensión profunda del conocimiento de los procesos constructivos, así como la comprensión de proyecto arquitectónico como un conjunto de sistemas constructivos integrados en un todo funcional, el cual fue modelado y vislumbrado antes de ser materializado en la obra.

\section{Recomendaciones}

Las posibles recomendaciones para seguir profundizando en el área temática de la investigación son las siguientes

- La implementación metodológica se realizó en un curso de tercer semestre por iniciativa de un grupo de profesores, es recomendable realizar exploraciones en cursos más altos, específicamente en construcciones de alta complejidad dado que el nivel de conocimiento de los estudiantes es mayor los resultados pueden ser excelentes.

- De acuerdo con los avances tecnológicos actuales donde la evolución es muy rápida, se debería poder planearse que se implemente esta metodología que inicialmente ha sido de carácter exploratoria se utilizara en los demás semestres, ajustándola a cada materia en particular en conjunto con los aportes de los decentes del área.

- Se sugiere la urgente necesidad de formar a de los profesores dado que como es natural existen resistencia al cambio en relación con la percepción de algunos docentes en cuanto al uso de estas nuevas tecnologías en las diversas áreas de arquitectura, donde estas pue-dan aportar.

- En el ámbito colombiano en el área de Diseño hay un rechazo a utilizar tecnologías de punta debido a que se piensa que se pierde el proceso creativo. Una posible forma sortear al rechazo mencionado en el ítem anterior es la generación de electivas que promuevan el uso de las tecnologías digitales de punta, pero con un énfasis en la parte creativa. 
- Esta investigación se desarrolló en algunos cursos, por interés de un grupo de profesores, sería adecuado que fuese liderado y apoyado por las directivas de la facultad, con el objetivo generar mejores perfiles de competitividad en la formación nuevas generaciones de estudiantes de arquitectura.

\section{Referencias}

Andrade R. (2021). Análisis en la variación en cantidades de obra y presupuesto entre la metodología tradicional y metodología BIM, caso de estudio: edificio de carrera de arquitectura. UNACH. 165 hojas. Quito: EPN.

Arrieta E. compiladora (2018). Memorias del Seminario la Representación del proyecto la Representación del Territorio. cuarta edición, disponible en Consultado el 7 de marzo del $2021 \mathrm{https}$ //arquitectura.medellin.unal.edu.co/images/documentos/MEMORIAS.pdf.

Biggs, J. (2006) Calidad del aprendizaje universitario. Madrid: Narcea S. A. Ediciones

Cabero J. \& Costas J. (2017) Simulators use for students training. Prisma Social No17 sección abierta pp. 343-372. Universidad de Sevilla, España https://www.redalyc.org/ pdf/3537/353749552015.pdf

Chávez E. (2018). Entornos de educación virtual 3d como estrategias de aprendizaje Tesis de maestría, Facultad de Ciencias Humanas y de la Educación, Maestría en Informática Educativa. Ambato Ecuador.

Espinoza M. C. (2016). Desarrollo de la competencia profesional basado en principios de Merrill. 135-148. Horizonte de la Ciencia 6 (11) diciembre 2016 FE/UNCP. ISSN (Impreso): 23044330/ ISSN (En Línea): 2413-936X Espinoza, C.

Guitert, M. \& Giménez, F. (2005). Trabajo cooperativo en entornos virtuales de aprendizaje. En J. Duart y A. Sangrá. (Eds.), Aprender en la virtualidad: 113-133. Barcelona: Editorial Gedisa, S. A.

Honebein P. \& Reigeluth Charles M. (2020). The instructional theory framework appears lost. Isn't it time we find it again? RED. Revista de Educación a Distancia. Núm. 64, Vol. 2 0. Artículo. $1,30-09-2020$

Jonassen D. (2000). El diseño de entornos constructivistas de aprendizaje EAC. En Ch. Reigeluth (Eds.), Diseño de la instrucción. Teorías y modelos. Un nuevo paradigma de la teoría de la instrucción. Parte I: 225-249. Madrid: Editorial Aula XXI Santillana.

Jonassen, nombrado por Chávez E. 2018 El constructivismo se enfoca en la preparación del que aprende para resolver problemas en condiciones complejas.

López F, Cubillos S, Gómez F, Escobar N, Cortés E \& Salcedo M. (2012). Modelación digital para el aprendizaje de la integración de sistemas en procesos constructivos. Bogotá: Universidad Nacional de Colombia.

López, F. \& Cubillos, S. (2011) Procesos de aprendizaje en coordinación técnica de la construcción mediados por simulación digital. Segundo Congreso Internacional Uso y Buenas Prácticas con TIC. Volumen 1. Málaga, España. Facultad de Ciencias de la Educación. Universidad 
de Málaga.

López, F., Cubillos, S., Maury, M. Gómez, F. \& Escobar, N. (2016). Evaluación de la calidad educativa en multimedios interactivos. En G. Hernández y F. Vargas (Eds.). Experiencias significativas en innovación pedagógica: 202-216. Bogotá: Universidad Nacional de Colombia.

Merritt, F. \& Ricketts, J. (2002) Building Design and Construction Handbook, 6th Edition McGraw -Hill Professional .

Orbe M, Toledo J \& Avila M. 2021. Analysis of BIM Methodologies Applied to Construction SMEs. Case Study: Cuenca- Ecuador. International Journal of Innovative Science and Research Technology Volume 6, Issue 2, February - 2021.

Rodríguez, C. \& Fernández-Batanero, J. (2017). Evaluación del Aprendizaje Basado en Problemas en Estudiantes Universitarios de construcción. Formación universitaria, 10(1), 61-70. Consultado el 13/03/2021. Disponible en https://dx.doi.org/10.4067/S071850062017000100007.

Sampaio, A.Z. \& Gomes, A.M. (2021) BIM Interoperability Analyses in Structure Design. Civil Eng., 2, 174-192. https://doi.org/10.3390/civileng2010010 